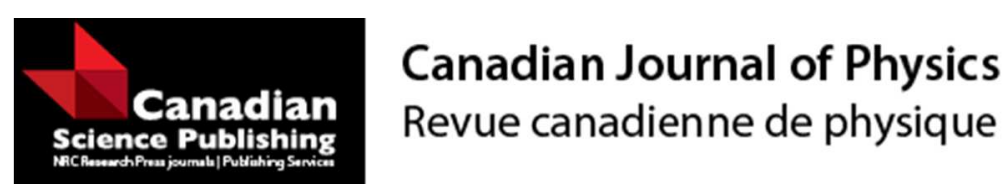

\title{
Symmetric Bound States of Josephson Vortices in BEC
}

\begin{tabular}{|r|l|}
\hline Journal: & Canadian Journal of Physics \\
\hline Manuscript ID & cjp-2017-0269.R1 \\
\hline Manuscript Type: & Article \\
\hline Complete List of Authors: & $\begin{array}{l}\text { Qadir, Muhammad; University of Engineering \& Technology Lahore, } \\
\text { Mathematics } \\
\text { Zoma, Tehseen; University of Engineering and Technology, Lahore, } \\
\text { Mathematics }\end{array}$ \\
\hline Keyword: & $\begin{array}{l}\text { Soliton, Bose-Einstein condensate, Josephson junction, Josephson vortices, } \\
\text { Bound states }\end{array}$ \\
\hline $\begin{array}{r}\text { Is the invited manuscript for } \\
\text { consideration in a Special } \\
\text { Issue? : }\end{array}$ & N/A \\
\hline
\end{tabular}




\title{
Symmetric Bound States of Josephson Vortices in BEC
}

\author{
Muhammad Irfan Qadir and Tehseen Zoma \\ Department of Mathematics, University of Engineering \& Technology, \\ Lahore 54890, Pakistan
}

Corresponding author: Muhammad Irfan Qadir; Email: mirfan@uet.edu.pk

PACS: 03.75.Lm, 03.75.Hh, 74.50.+r.

\begin{abstract}
A system of two parallel coupled cigar shaped Bose-Einstein condensates is considered in effectively one dimensional limit. The dynamics of the system is characterized by a pair of coupled nonlinear Gross-Pitaevskii equations. In particular, the existence and stability of symmetric bound states of Josephson vortices are investigated. It is realized that the symmetric bound state Josephson vortices solution persists stably in its whole domain of existence for the coupling strength. Nevertheless, the bound states solution converts into a dark soliton at a critical value of coupling parameter.
\end{abstract}

Keywords: Soliton, Bose-Einstein condensate, Josephson junction, Josephson vortices, Bound states.

\section{INTRODUCTION}

Bose-Einstein condensate (BEC) is one of the captivating states of matter that was first predicted by Satyendra Nath Bose and Albert Einstein in 1924 [1, 2, 3]. It was experimentally created by the team led by Wieman and Cornell using $R b^{87}$ at Joint Institute for Laboratory Astrophysics (JILA) in July 1995 [4]. W. Ketterle and his colleagues realized BEC using sodium in Massachusetts Institute of Technology (MIT) in November 1995 [5].

$\mathrm{BEC}$ has created a lot of interest in nonlinear matter waves such as bright solitons [6, 7], dark solitons $[8,9]$ and vortices $[10,11]$. In BEC, bright solitons are emerged due to attractive interactions while dark solitons due to repulsive interactions of atoms. In one dimension, dark solitons are stable particle like objects [12] but in higher dimension they possess snake instability [13]. In spherical condensates, instabilities stimulus the dark solitons to slide into stable vortex rings.

The concept of Bose-Josephson junction which is analogues to superconducting Josephson junction was proposed by Smerzi [14]. Josephson vortices (circulation of supercurrent) can exist in long BoseJosephson junction [15] which is formed between two parallel quasi one dimensional BEC coupled 
by tunneling. These vortices can be handled by applying a difference of chemical potential on the atomic BEC waveguide forming the junction $[16,17]$. Most interesting feature of Bose-Josephson vortex is its interconversion into dark soliton. In small Bose-Josephson junction [14, 18, 19], the presence of oscillations makes conversion from the superconducting state to the resistive state at an unusual value of the bias current $I$. In the long Bose-Josephson junction having length $\ell>\lambda_{j}$ (where $\lambda_{j}$ is the penetration depth), this conversion may be in the form of discharge of a Josephson fluxon from a pinning potential [20].

In connection with the study of parallel potential waveguide of coupled BEC in quasi one dimensional limit, the existence and stability of Josephson vortices and their transformation into coupled dark solitons and vice versa were presented in $[16,17]$. The existence and stability analysis of these vortices and their conversion into coupled dark solitons under the influence of a harmonic potential was exhibited in $[21,22]$. Due to the symmetry of the imaginary parts of the bound state solutions, they can be classified into anti-symmetric bound state Josephson vortices and symmetric bound state Josephson vortices solutions. The study of existence and stability of anti-symmetric atomic bound states of Josephson vortices was presented in [23]. In case of nonlinear Schrodinger equation with parametric drive, the stability analysis of these bound states was presented in [24, 25] where these solutions corresponds to Bloch-Bloch states.

The purpose of this paper is to take into account the symmetric bound states of Josephson vortices in two parallel one dimensional coupled BEC. To the best of our knowledge, the existence and stability of these symmetric bound states have not been investigated yet. In particular, we study the effects of variation of coupling strength on the stability of the bound states.

We organize the paper as follows. In Section 2, we introduce the model and find the time independent symmetric bound states of Josephson vortices numerically. In Section 3, we present the linear stability analysis of these bound states using Bogoliubov-de Gennes (BdG) equations. For the verification of the results obtained, we numerically solve the coupled Gross-Pitaevskii equations. We conclude our results in Section 4.

\section{MATHEMATICAL MODEL AND COMPUTATIONAL SETUP}

For a condensed system in which all particles are in the same quantum state, BEC is well described by a wave function governed by the mean field theory. The time dependent Gross-Pitaevskii equation is the model equation which is used for the wave function describing the quantum state of particles. The coupled Gross-Pitvaeskii equation with identical chemical potential $\omega$ is given as 


$$
\begin{aligned}
& \frac{i \partial}{\partial t} A_{1}(x, t)=-\frac{1}{2} \frac{\partial^{2}}{\partial x^{2}} A_{1}(x, t)+g\left|A_{1}(x, t)\right|^{2} A_{1}(x, t)-\omega A_{1}(x, t)+\gamma A_{2}(x, t), \\
& \frac{i \partial}{\partial t} A_{2}(x, t)=-\frac{1}{2} \frac{\partial^{2}}{\partial x^{2}} A_{2}(x, t)+g\left|A_{2}(x, t)\right|^{2} A_{2}(x, t)-\omega A_{2}(x, t)+\gamma A_{1}(x, t),
\end{aligned}
$$

where $A_{1}(x, t)$ and $A_{2}(x, t)$ are the wave functions describing the densities of the condensates in the mean field. $\gamma$ is the coupling strength and $g=\frac{4 \pi \hbar^{2} a_{s}}{m}$ (where $m$ is the mass of the particles of the condensate and $a_{s}$ is the scattering length) is the nonlinear interaction between particles in BEC which can either be attractive or repulsive. We have considered the interatomic interaction to be positive i.e. $g>0$. For the time independent solution, we substitute

$$
\frac{\partial A_{1}}{\partial t}=0=\frac{\partial A_{2}}{\partial t}
$$

So that eq. (1) and eq. (2) become

$$
\begin{aligned}
& -\frac{1}{2} \frac{\partial^{2} A_{1}(x, t)}{\partial x^{2}}+g\left|A_{1}(x, t)\right|^{2} A_{1}(x, t)-\omega A_{1}(x, t)+\gamma A_{2}(x, t)=0 \\
& -\frac{1}{2} \frac{\partial^{2} A_{2}(x, t)}{\partial x^{2}}+g\left|A_{2}(x, t)\right|^{2} A_{2}(x, t)-\omega A_{2}(x, t)+\gamma A_{1}(x, t)=0 .
\end{aligned}
$$

To discretize eq. (4) and eq. (5), we use central difference formulas for the second derivative, so that

$$
\begin{aligned}
& -\frac{1}{2}\left(\frac{A_{1, n+1}+A_{1, n-1}-2 A_{1, n}}{h^{2}}\right)+g\left|A_{1, n}\right|^{2} A_{1, n}-\omega A_{1, n}+\gamma A_{2, n}=0, \\
& -\frac{1}{2}\left(\frac{A_{2, n+1}+A_{2, n-1}-2 A_{2, n}}{h^{2}}\right)+g\left|A_{2, n}\right|^{2} A_{2, n}-\omega A_{2, n}+\gamma A_{1, n}=0 .
\end{aligned}
$$

Using Neumann boundary conditions

$$
\begin{aligned}
& \left.\frac{\partial A_{1}}{\partial x}\right|_{x= \pm L}=0 \\
& \left.\frac{\partial A_{2}}{\partial x}\right|_{x= \pm L}=0
\end{aligned}
$$

we get a system of nonlinear equations where each coordinate function $f_{l}(l=1,2,3, \ldots, 2 \mathrm{~N})$ is mapping a vector $\boldsymbol{A}=\left(A_{1,1}, A_{1,2}, \ldots A_{1, N}, A_{2,1}, A_{2,2}, \ldots A_{2, N}\right)^{t}$ of $2 N$-dimensional space $\mathfrak{N}^{2 N}$ into the real line $\mathfrak{N}$. The system of nonlinear equations can be represented as 


$$
\boldsymbol{F}(\boldsymbol{A})=\left(f_{1}(\boldsymbol{A}), f_{2}(\boldsymbol{A}), \ldots, f_{2 N}(\boldsymbol{A})\right)^{t}=0
$$

To solve the system $\boldsymbol{F}(\boldsymbol{A})=0$, we construct the algorithm that leads to a fixed point for the $2 \mathrm{~N}$ dimensional case which involves a matrix

$$
M(\boldsymbol{A})=\left[\begin{array}{cccc}
m_{1,1}(\boldsymbol{A}) & m_{1,2}(\boldsymbol{A}) & \cdots & m_{1,2 N}(\boldsymbol{A}) \\
m_{2,1}(\boldsymbol{A}) & m_{2,2}(\boldsymbol{A}) & \cdots & m_{2,2 N}(\boldsymbol{A}) \\
\vdots & \vdots & \ddots & \vdots \\
m_{2 N, 1}(\boldsymbol{A}) & m_{2 N, 2}(\boldsymbol{A}) & \cdots & m_{2 N, 2 N}(\boldsymbol{A})
\end{array}\right]
$$

where each of the entries $m_{l, p}(\boldsymbol{A}), l, p=1,2,3, \ldots, 2 N$ is a function from $\mathfrak{R}^{2 N}$ into $\mathfrak{R}$. This requires that $M(A)$ is to be found so that

$$
\boldsymbol{Q}(\boldsymbol{A})=\boldsymbol{A}-M(\boldsymbol{A})^{-1} \boldsymbol{F}(\boldsymbol{A})
$$

gives quadratic convergence to the solution of $\boldsymbol{F}(\boldsymbol{A})=0$ with the assumption that $M(\boldsymbol{A})$ is nonsingular at the fixed point $\boldsymbol{q}$ of $\boldsymbol{Q}$. For this purpose, we use the following theorem [26].

\section{Theorem:}

Let $\boldsymbol{q}$ be a solution of $\boldsymbol{Q}(\boldsymbol{A})=\boldsymbol{A}$ and suppose that there exists a number $\delta>0$ with

(i) $\frac{\partial q_{i}}{\partial \boldsymbol{A}_{j, k}}$ is continuous on $N_{\delta}=\{\boldsymbol{A} \mid\|\boldsymbol{A}-\boldsymbol{q}\|<\delta\}$, for each $i=1,2, \ldots, 2 N, j=1,2, k=1,2, \ldots, N$,

(ii) $\frac{\partial^{2} q_{i}(\boldsymbol{A})}{\partial A_{j, k} \partial A_{j, r}}$ is continuous and $\left|\frac{\partial^{2} q_{i}(\boldsymbol{A})}{\partial A_{j, k} \partial A_{j, r}}\right| \leq M$ for some constant $\mathrm{M}$, whenever $\boldsymbol{A} \in N_{\delta}$, for $i=$ $1,2, \ldots, 2 N, j=1,2, k=1,2, \ldots, N$, and $r=1,2, \ldots, N$,

(iii) $\frac{\partial q_{i}(\boldsymbol{q})}{\partial A_{j, r}}=0$ for each $i=1,2,3 \ldots, 2 N, j=1,2$ and $r=1,2, \ldots, N$.

Then there exists a number $\delta^{\prime} \leq \delta$ such that the sequence generated by $\boldsymbol{A}^{(\boldsymbol{s})}=\boldsymbol{Q}\left(\boldsymbol{A}^{(\boldsymbol{s}-1)}\right)$ converges quadratically to $\boldsymbol{q}$ for any choice of $\boldsymbol{A}^{(0)}$, provided that $\left\|\boldsymbol{A}^{(0)}-\boldsymbol{q}\right\| \leq \delta^{\prime}$.

To apply the above theorem, we suppose that $M(\boldsymbol{A})$ in (10) is non-singular near a solution $\boldsymbol{q}$ of $\boldsymbol{F}(\boldsymbol{A})=0$. Let $b_{l, p}(\boldsymbol{A})$ be the elements of $M(\boldsymbol{A})^{-\mathbf{1}}$ in the $l$ th row and $p$ th column.

For $\boldsymbol{Q}(\boldsymbol{A})=\boldsymbol{A}-M(\boldsymbol{A})^{-1} \boldsymbol{F}(\boldsymbol{A})$, we have

$$
q_{l}(\boldsymbol{A})=A_{j, l}-\sum_{p=1}^{2 N} b_{l, p}(\boldsymbol{A}) f_{p}(\boldsymbol{A})
$$


for each $l=1,2, \ldots, 2 N$ and $j=1,2$.

When $j=1$ and $l=1,2, \ldots, N$, we have

$$
\frac{\partial q_{l}(\boldsymbol{A})}{\partial A_{1, l}}= \begin{cases}1-\sum_{p=1}^{2 N}\left(b_{l, p}(\boldsymbol{A}) \frac{\partial f_{p}(\boldsymbol{A})}{\partial A_{1, l}}+\frac{\partial b_{l, p}(\boldsymbol{A})}{\partial A_{1, l}} f_{p}(\boldsymbol{A})\right), & l=1 \\ -\sum_{p=1}^{2 N}\left(b_{l, p}(\boldsymbol{A}) \frac{\partial f_{p}(\boldsymbol{A})}{\partial A_{1, l}}+\frac{\partial b_{l, p}(\boldsymbol{A})}{\partial A_{1, l}} f_{p}(\boldsymbol{A})\right), & l \neq 1\end{cases}
$$

When $j=2$ and $l=N+1, N+2, \ldots, 2 N$, we have

$$
\frac{\partial q_{i}(\boldsymbol{A})}{\partial A_{2, l}}= \begin{cases}1-\sum_{p=N+1}^{2 N}\left(b_{l, p}(\boldsymbol{A}) \frac{\partial f_{l}(\boldsymbol{A})}{\partial A_{2, l}}+\frac{\partial b_{l, p}(\boldsymbol{A})}{\partial A_{2, l}} f_{p}(\boldsymbol{A})\right), & l=N+1 \\ -\sum_{p=N+1}^{2 N}\left(b_{l, p}(\boldsymbol{A}) \frac{\partial f_{l}(\boldsymbol{A})}{\partial A_{2, l}}+\frac{\partial b_{l, p}(\boldsymbol{A})}{\partial A_{2, l}} f_{p}(\boldsymbol{A})\right), & l \neq N+1\end{cases}
$$

According to condition (iii) of the above theorem, we need $\frac{\partial q_{l}(\boldsymbol{q})}{\partial \boldsymbol{A}_{j, l}}=0$ for $j=1,2, l, p=1,2, \ldots, 2 N$.

So, for $j=1$ and $l=1$, we have

$$
\sum_{p=1}^{2 N} b_{l, p}(\boldsymbol{q}) \frac{\partial f_{p}(\boldsymbol{q})}{\partial A_{1, l}}=1
$$

and for $1<l \leq N$, we have

$$
\sum_{p=1}^{2 N} b_{l, p}(\boldsymbol{q}) \frac{\partial f_{p}(\boldsymbol{q})}{\partial A_{1, l}}=0
$$

Similarly, for $j=2$ and $l=N+1$, we have

$$
\sum_{p=1}^{2 N} b_{l . p}(\boldsymbol{q}) \frac{\partial f_{p}(\boldsymbol{q})}{\partial A_{2, l}}=1
$$

and for $N+1<l \leq 2 N$, we have

$$
\sum_{p=1}^{2 N} b_{l, p}(\boldsymbol{q}) \frac{\partial f_{p}(\boldsymbol{q})}{\partial A_{2, l}}=0
$$


Using equations (14), (15), (16) and (17), one can find the entries of $M(\boldsymbol{A})^{\mathbf{- 1}}$. An iteration procedure evolves from selecting $\boldsymbol{A}^{(0)}$ and generating for $s \geq 1$

$$
\boldsymbol{A}^{(s)}=\boldsymbol{Q} \boldsymbol{A}^{(s-1)}=\boldsymbol{A}^{(s-1)}-M\left(\boldsymbol{A}^{(s-1)}\right)^{-1} \boldsymbol{F}\left(\boldsymbol{A}^{(s-1)}\right)
$$

We use the above algorithm to find the symmetric bound states of Josephson vortices. The solution obtained is shown in Fig. 1.

\section{STABILITY OF SYMMETRIC BOUND STATE SOLUTION}

Now we discuss the stability of the symmetric bound states of atomic Josephson vortices. Suppose $A_{1,2}^{(0)}$ be the steady state solutions of the coupled Gross-Pitaevskii equations. Let us consider small perturbations $v_{1}, v_{2}$ in the solutions such that $v_{1}, v_{2} \ll 1$, so that

$$
\begin{aligned}
& A_{1}(x, t)=A_{1}^{(0)}+v_{1}(x, t), \\
& A_{2}(x, t)=A_{2}^{(0)}+v_{2}(x, t) .
\end{aligned}
$$

Substituting these values into eq. (1) and eq. (2), we obtain

$$
\begin{aligned}
& \frac{i \partial v_{1}}{\partial t}=-\frac{1}{2}\left(\frac{\partial^{2} A_{1}^{(0)}}{\partial x^{2}}+\frac{\partial^{2} v_{1}}{\partial x^{2}}\right)+g\left|A_{1}^{(0)}+v_{1}\right|^{2}\left(A_{1}^{(0)}+v_{1}\right)-\omega\left(A_{1}^{(0)}+v_{1}\right)+\gamma\left(A_{2}^{(0)}+v_{2}\right), \\
& \frac{i \partial v_{2}}{\partial t}=-\frac{1}{2}\left(\frac{\partial^{2} A_{2}^{(0)}}{\partial x^{2}}+\frac{\partial^{2} v_{2}}{\partial x^{2}}\right)+g\left|A_{2}^{(0)}+v_{2}\right|^{2}\left(A_{2}^{(0)}+v_{2}\right)-\omega\left(A_{2}^{(0)}+v_{2}\right)+\gamma\left(A_{1}^{(0)}+v_{1}\right) .
\end{aligned}
$$

As $v_{1}, v_{2}$ are very small, by neglecting their higher terms, we get

$$
\begin{aligned}
& \frac{i \partial v_{1}}{\partial t}=-\frac{1}{2}\left(\frac{\partial^{2} v_{1}}{\partial x^{2}}\right)+g\left(A_{1}^{(0)}\right)^{2} \overline{v_{1}}+2 g\left|A_{1}^{(0)}\right|^{2} v_{1}-\omega v_{1}+\gamma v_{2}, \\
& \frac{i \partial v_{2}}{\partial t}=-\frac{1}{2}\left(\frac{\partial^{2} v_{2}}{\partial x^{2}}\right)+g\left(A_{2}^{(0)}\right)^{2} \overline{v_{2}}+2 g\left|A_{2}^{(0)}\right|^{2} v_{2}-\omega v_{2}+\gamma v_{1} .
\end{aligned}
$$

We take the complex conjugate of the above equations (23) and (24), the resulting equations can be written as

$$
\begin{aligned}
& \frac{i \partial \overline{v_{1}}}{\partial t}=\frac{1}{2}\left(\frac{\partial^{2} \overline{v_{1}}}{\partial x^{2}}\right)-g \overline{\left(A_{1}^{(0)}\right)^{2}} v_{1}-2 g\left|A_{1}^{(0)}\right|^{2} \overline{v_{1}}+\omega \overline{v_{1}}-\gamma \overline{v_{2}} \\
& \frac{i \partial \overline{v_{2}}}{\partial t}=\frac{1}{2}\left(\frac{\partial^{2} \overline{v_{2}}}{\partial x^{2}}\right)-g \overline{\left(A_{2}^{(0)}\right)^{2}} v_{2}-2 g\left|A_{2}^{(0)}\right|^{2} \overline{v_{2}}+\omega \overline{v_{2}}-\gamma \overline{v_{1}}
\end{aligned}
$$


where bar is the notation used for the complex conjugation. For simplicity, we write $v_{1}=a, v_{2}=b, \overline{v_{1}}=$ $c, \overline{v_{2}}=d$, so that the above equations can be written as

$$
\begin{aligned}
& \frac{i \partial a}{\partial t}=-\frac{1}{2}\left(\frac{\partial^{2} a}{\partial x^{2}}\right)+g\left(A_{1}^{(0)}\right)^{2} c+2 g\left|A_{1}^{(0)}\right|^{2} a-\omega a+\gamma b=\Lambda a, \\
& \frac{i \partial b}{\partial t}=-\frac{1}{2}\left(\frac{\partial^{2} b}{\partial x^{2}}\right)+g\left(A_{2}^{(0)}\right)^{2} d+2 g\left|A_{2}^{(0)}\right|^{2} b-\omega b+\gamma a=\Lambda b, \\
& \frac{i \partial c}{\partial t}=\frac{1}{2}\left(\frac{\partial^{2} c}{\partial x^{2}}\right)-g \overline{\left(A_{1}^{(0)}\right)^{2}} a-2 g\left|A_{1}^{(0)}\right|^{2} c+\omega c-\gamma d=\Lambda c, \\
& \frac{i \partial d}{\partial t}=\frac{1}{2}\left(\frac{\partial^{2} d}{\partial x^{2}}\right)-g \overline{\left(A_{2}^{(0)}\right)^{2} b}-2 g\left|A_{2}^{(0)}\right|^{2} d+\omega d-\gamma c=\Lambda d,
\end{aligned}
$$

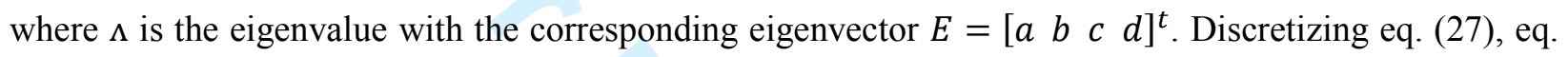
(28), eq. (29) and eq. (30), we obtain an eigenvalue problem $\xi E=\Lambda E$, in which

$$
\begin{aligned}
& \xi=\left[\begin{array}{cccc}
\alpha_{1} & \beta_{1} & \Gamma & 0 \\
\Gamma & O & \alpha_{2} & \beta_{2} \\
-\bar{\beta}_{1} & -\alpha_{1} & 0 & -\Gamma \\
0 & -\Gamma & -\bar{\beta}_{2} & \alpha_{2}
\end{array}\right], \\
& \Gamma=\left[\begin{array}{ccccc}
\gamma & 0 & 0 & \cdots & 0 \\
0 & \gamma & 0 & \cdots & 0 \\
0 & 0 & \gamma & \cdots & 0 \\
\vdots & \vdots & \vdots & \ddots & \vdots \\
0 & 0 & 0 & \cdots & \gamma
\end{array}\right] \\
& \beta_{1}=\left[\begin{array}{ccccc}
g\left(A_{1,1}^{(0)}\right)^{2} & 0 & 0 & \cdots & 0 \\
0 & g\left(A_{1,2}^{(0)}\right)^{2} & 0 & \cdots & 0 \\
0 & 0 & g\left(A_{1,3}^{(0)}\right)^{2} & \cdots & 0 \\
\vdots & \vdots & \vdots & \ddots & \vdots \\
0 & 0 & 0 & \cdots & g\left(A_{1, N}^{(0)}\right)^{2}
\end{array}\right],
\end{aligned}
$$




$$
\beta_{2}=\left[\begin{array}{ccccc}
g\left(A_{2,1}^{(0)}\right)^{2} & 0 & 0 & \cdots & 0 \\
0 & g\left(A_{2,2}^{(0)}\right)^{2} & 0 & \cdots & 0 \\
0 & 0 & g\left(A_{2,3}^{(0)}\right)^{2} & \cdots & 0 \\
\vdots & \vdots & \vdots & \ddots & \vdots \\
0 & 0 & 0 & \cdots & g\left(A_{2, N}^{(0)}\right)^{2}
\end{array}\right]
$$

$$
\begin{aligned}
& \dot{\alpha}_{1}=\left[\begin{array}{ccccc}
\frac{1}{2 h^{2}}+2 g\left|A_{1,1}^{(0)}\right|^{2}-\omega & -\frac{1}{2 h^{2}} & 0 & \cdots & 0 \\
-\frac{1}{2 h^{2}} & \frac{1}{h^{2}}+2 g\left|A_{1,2}^{(0)}\right|^{2}-\omega & -\frac{1}{2 h^{2}} & \cdots & 0 \\
\vdots & \vdots & \vdots & \ddots & \vdots \\
0 & 0 & 0 & \cdots & \frac{1}{2 h^{2}}+2 g\left|A_{1, N}^{(0)}\right|^{2}-\omega
\end{array}\right] \text {, } \\
& \dot{\alpha}_{2}=\left[\begin{array}{ccccc}
\frac{1}{2 h^{2}}+2 g\left|A_{2,1}^{(0)}\right|^{2}-\omega & -\frac{1}{2 h^{2}} & 0 & \cdots & 0 \\
-\frac{1}{2 h^{2}} & \frac{1}{h^{2}}+2 g\left|A_{2,2}^{(0)}\right|^{2}-\omega & -\frac{1}{2 h^{2}} & \cdots & 0 \\
\vdots & \vdots & \vdots & \ddots & \vdots \\
0 & 0 & 0 & \cdots & \frac{1}{2 h^{2}}+2 g\left|A_{2, N}^{(0)}\right|^{2}-\omega
\end{array}\right] .
\end{aligned}
$$

We find the eigenvalues of matrix $\xi$ for investigating the stability of the symmetric bound states of Josephson vortices. The solution will explicit be stable when all eigenvalues are real. In Fig. (2), on horizontal axis we have taken the real parts and on vertical axis we have taken the imaginary parts of the eigenvalues. From the figure, it is clear that all eigenvalues are lying horizontally and show the stability of the symmetric bound states of Josephson vortices solution.

In order to investigate the stability of the symmetric bound states of Josephson vortices for different values of coupling constant $\gamma$, we plot the graph of $\gamma$ versus the maximum value of the imaginary parts of the eigenvalues. It is noticed that the maximum of the imaginary parts of all eigenvalues remain zero in whole of the domain of existence of symmetric solution for the coupling parameter as shown by red line in Fig. 3. However, the amplitudes of the humps of the imaginary parts decrease with the increase in the value of $\gamma$. The humps become flat at $\gamma=1 / 3$ showing that the bound states solution has changed into a coupled dark soliton solution. The dark soliton solution remains unstable for $\gamma<1 / 3$ but changes stably 
into a dark soliton and remains stable for $\gamma \geq 1 / 3$ as depicted in Fig. 3 by green curve. We also noticed that $\gamma=1 / 3$ is the point of bifurcation for the solutions. The bifurcation in this case is a pitchfork bifurcation as shown in Fig. 4. The figure shows the domain of existence of the dark soliton as well as the symmetric bound state Josephson vortices solutions.

For the verification of the above results, we numerically integrate the coupled system of equations (1) and (2). In particular, we employ the fourth order Runge-Kutta method and used the static symmetric bound state solution as the initial condition. The solution is found to stable and justifies the results obtained. However, the dark soliton solution is found to be unstable in the domain of existence of symmetric bound state Josephson vortices solution. The time evolution of the dark soliton solution is depicted in Fig. 5 showing the instability of the dark soliton solution. Due to instability, radiations emerge near $t=30$.

\section{CONCLUDING REMARKS}

The aim of this paper was to investigate the existence and stability of symmetric bound state Josephson vortices solution in two parallel quasi one-dimensional cigar shaped coupled BEC. Our analysis was based on a system of two coupled Gross-Pitaevskii equations. It has been found that the symmetric bound state Josephson vortices solution exists for $0<\gamma<1 / 3$. The stability of the solution has been examined by the variation in the value of coupling parameter. The solution was found to be stable in its entire domain where it exists. The bound state solution changed into a coupled dark soliton solution at $\gamma=1 / 3$ which was a bifurcation point and the bifurcation was a pitchfork bifurcation. The coupled dark soliton solution persisted and remained unstable for $\gamma<1 / 3$ but became stable for $\gamma \geq 1 / 3$.

\section{REFERENCES}

[1] L. P. Pitvaeskii and S. Stringari. Bose-Einstein condensation and superfluidity, Oxford University Press, UK (2016).

[2] P.G. Kevrekidis, D. J. Frantzeskakis and R. Carretero-Gonz'alez. Emergent nonlinear phenomena in BoseEinstein condensates, Springer-Verlag Berlin Heidelberg (2008).

[3] C. J. Pethick and H. Smith. Bose-Einstein condensation in dilute gases, Cambridge University Press, UK (2008).

[4] M. H. Anderson, J. R. Ensher, M. R. Matthews, C. E. Wieman and E. A. Cornell. Observation of BoseEinstein condensation in a dilute atomic vapor, Science 269, 198-201 (1995).

[5] K. B. Davis, M. O. Mewes, M. R. Andrews, N. J. van Druten, D. S. Durfee, D. M. Kurn and W. Ketterle. Bose-Einstein condensation in a gas of sodium atoms, Physical Review Letters 75, 3969-3973 (1995). 
[6] K. E. Strecker, G. B. Partridge, A. G. Truscott and R. G. Hulet. Formation and propagation of matter-wave soliton trains, Nature 417, 150-153 (2002).

[7] L. Khaykovich, F. Schreck, G. Ferrari, T. Bourdel, J. Cubizolles, L. D. Carr, Y. Castin and C. Salomon. Formation of a matter-wave bright soliton, Science 296, 1290-1293 (2002).

[8] S. Burger, K. Bongs, S. Dettmer, W. Ertmer, K. Sengstock, A. Sanpera, G. V. Shlyapnikov and M. Lewenstein. Dark solitons in Bose-Einstein condensates, Physical Review Letters 83, 5198-5201 (1999).

[9] D. J. Frantzeskakis. Dark solitons in atomic Bose-Einstein condensates, Journal of Physics A, Mathematical and Theoretical 43, 213001-213082 (2010).

[10] B. P. Anderson, P. C. Haljan, C. A. Regal, D. L. Feder, L. A. Collins, C. W. Clark, and E. A. Cornell. Watching dark solitons decay into vortex rings in a Bose-Einstein condensate, Physical Review Letters 86, 2926-2929 (2001).

[11] A. L. Fetter. Rotating trapped Bose-Einstein condensates, Reviews of Modern Physics, 81, 647-691. (2013).

[12] P. O. Fedichev, A. E. Muryshev and G. V Shlyapnikov, Dissipative dynamics of a kink state in a Bosecondensed gas, physical Review A 60, 3220-3234 (1999).

[13] A. E. Muryshev, H. B. van Linden van den Heuvell and G. V. Shlyapnikov, Stability of standing matter waves in a trap, physical Review A 60, R2665 (1999).

[14] A. Smerzi, S. Fantoni, S. Giovanazzi and S. R. Shenoy. Quantum coherent atomic tunneling between two trapped Bose-Einstein condensates, Physical Review Letters 79, 4950-4953 (1997).

[15] A. Barone and G. Paterno. Physics and Applications of the Josephson Effect, Wiley, New York. (1982).

[16] V. M. Kaurov and A. B. Kuklov, Josephson vortex between two atomic Bose-Einstein condensates, Physical Review A 71, 011601-011604 (2005).

[17] V. M. Kaurov and A. B. Kuklov, Atomic Josephson vortices, Physical Review A 73, 0136271-0136278 (2006).

[18] S. Giovanazzi, A. Smerzi and S. Fantoni. Josephson effects in dilute Bose-Einstein condensates, Physical Review Letters 84, 4521-4524 (2000).

[19] L. Pitaevskii and S. Stringari. Thermal vs quantum decoherence in double well trapped Bose-Einstein condensates, Physical Review Letters 87, 180402 (2001). 
[20] M. V. Fistual, M. G. Castellano, M. Cirillo, G. Torrioli, A. Wallraff and A. V. Ustinov. Escape of Josephson Vortex trapped in an annular Josephson junction, Physica B: Physics of Condensed Matter 284, 585$586(2000)$.

[21] M. I. Qadir, H. Susanto and P. C. Matthews. Journal of Physics B: Atomic, Molecular and Optical Physics 45, 035004 (2012).

[22] M. I. Qadir and N. Irshad. Proceeding of the Pakistan Academy of Sciences, A Physical and Computational Sciences 53: 17-24 (2016).

[23] M. I. Qadir and U. Tahir. Bound states of atomic Josephson vortices, Canadian Journal of Physics, 95: 336339 (2017).

[24] I. V. Barashenkov, S. R. Woodford and E. V. Zemlyanaya. Parametrically Driven Dark Solitons. Physical Review Letters 90: 054103 (2003).

[25] I. V. Barashenkov, S.R. Woodford and E. V. Zemlyanaya. Interactions of parametrically driven dark solitons. I. Néel-Néel and Bloch-Bloch interactions.Physical Review E 75: 026604 (2007).

[26] J. M. Ortega and W. C. Rheinboldt. Iterative solution of nonlinear equations in several variables, Academic Press, New York, 1970.
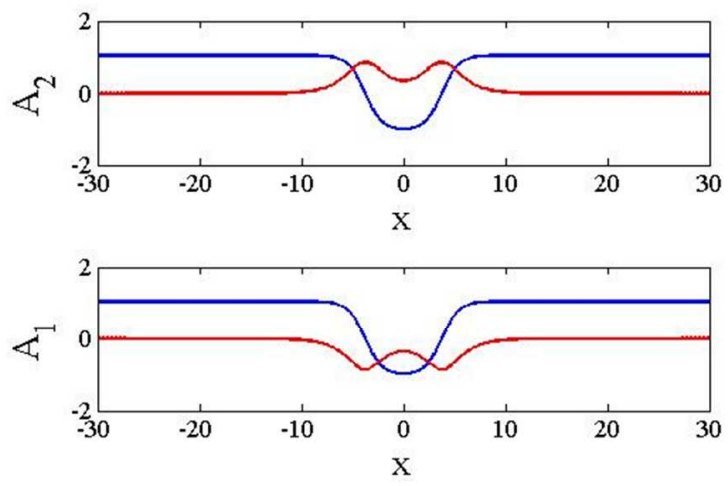

Fig. 1. The symmetric bound states Josephson vortices solution for $g=1, \omega=1$ and $\gamma=0.1$. The lower part corresponds to $A_{1}$ and the upper part to $A_{2}$. The red curves represent the real parts and the blues curves the imaginary parts of the solution. 


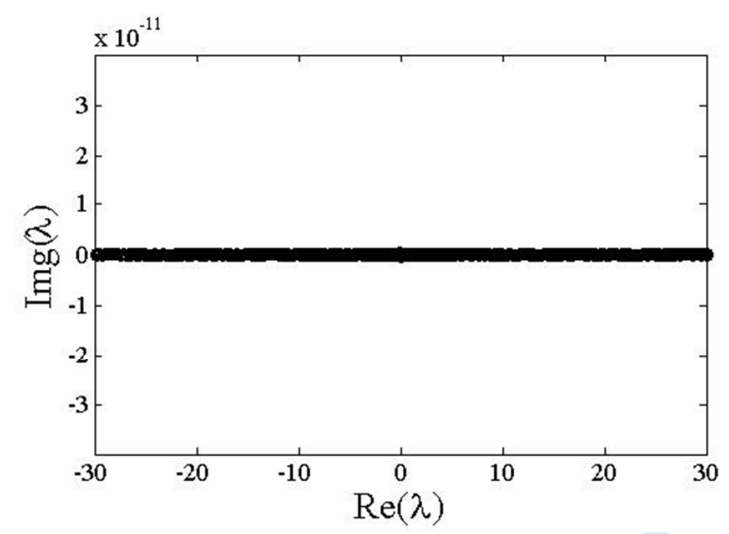

Fig. 2. The layout of eigenvalues for the symmetric bound states of Josephson vortices solution for $g=1, \omega=1$ and $\gamma=0.1$. All eigenvalues are lying horizontally showing that the solution is stable.

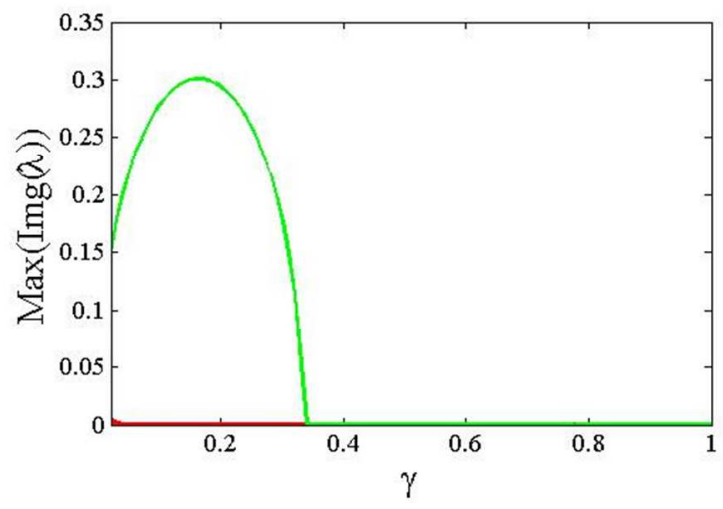

Fig. 3. The graph of coupling strength $\gamma$ versus the maximum value of the imaginary parts of the eigenvalues. The red line corresponds to the symmetric bound state solution and depicting that the solution is stable for all values of $\gamma$ while the green curve is for coupled dark soliton solution showing that the dark soliton solution attains stability for $\gamma \geq 1 / 3$. 


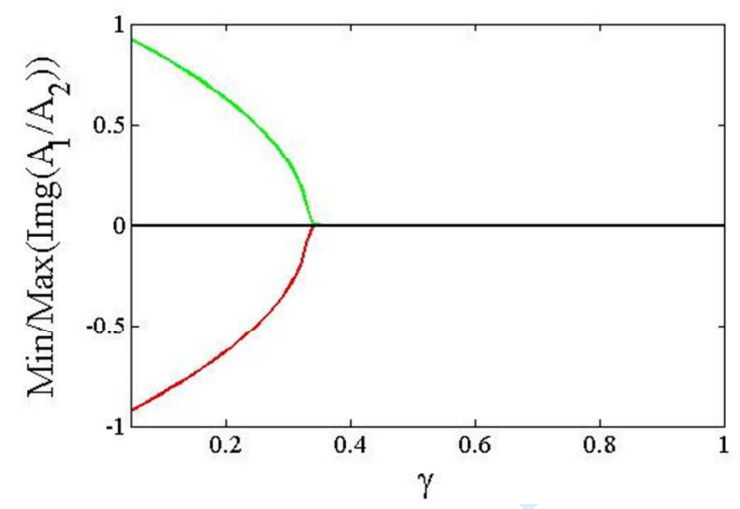

Fig.4. The amplitude of the humps of the imaginary parts of the solutions showing pitchfork bifurcation.
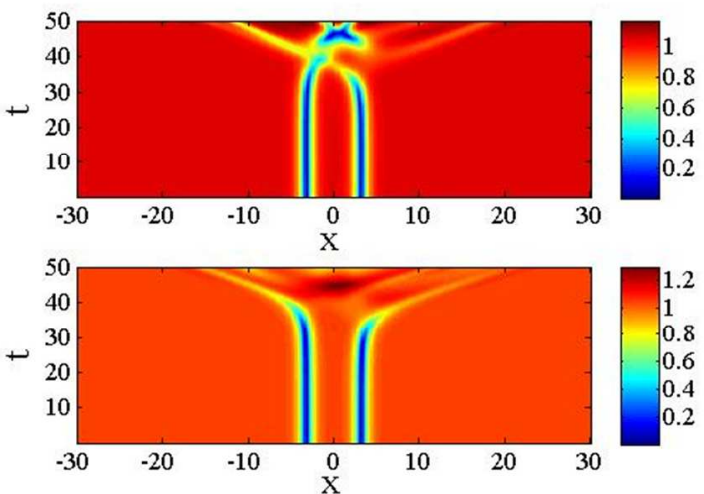

Fig.5. The contour plot for the time evolution of the coupled dark soliton for $g=1, \omega=1$ and $\gamma=0.1$. Radiation are evident near $t=30$ due to the instability of the solution. 